\title{
Law and Cartoons: La Sémiotique de Production et de Diffusion en Droit comme Stratégie de Communication
}

\author{
Anne Wagner ${ }^{1}$
}

Published online: 20 October 2015

(C) Springer Science+Business Media Dordrecht 2015

\begin{abstract}
Un jeu subtil associant les dimensions visuelles, culturelles ou sociales s'établit entre l'utilisateur de la règle et son destinataire. L'étude des différentes méthodes employées met en lumière cette dynamique du discours juridique. Cette nécessité de spécifier les rôles, de montrer les visages multiples a pour vocation de rendre sensible et conscient le locuteur au pluralisme organisé dans le discours juridique. C'est dans la multiplicité que le discours peut s'avérer fragile, susceptible de rupture dans la compréhension de sens.
\end{abstract}

Keywords Law $\cdot$ Culture $\cdot$ Visual studies $\cdot$ Cartoons $\cdot$ Semiotics

La sémiotique de production et de diffusion en droit permet la mise en évidence d'une dynamique dans la compréhension et l'assimilation du discours juridique. La sémiotique juridique insiste sur la contingence et la fluidité des concepts juridiques. Elle met également l'accent sur la présence de discours juridiques co-existants, concurrents et se chevauchant. Les doctrines et les pratiques sont en évolution constante par les mutations voulues des producteurs de règles mais également par la pression sociale. Les structures intermédiaires d'administration du droit jouent également un rôle important et complexe dans la formalisation, soit directement par le filtre d'identification technique propre au service, soit par l'effet de structure dans la diffusion du message. Il se dessine alors des hypothèses de travail concurrentes aux discours originels. Cette insécurité juridique pose la question de l'interprétation et confère au sémioticien un rôle d'observateur; ses recherches s'orientent en particulier sur les acteurs en charge de définir les mots et concepts utilisés.

Anne Wagner

valwagnerfr@yahoo.com

$1 \quad$ Lille, France 
Cet axe dans les «stratégies de communication en droit» permet aux linguistes, sémioticiens et juristes de s'attacher à confronter le «signe» dans la relation courante et l'application qui en est faite par les pouvoirs construits, et de tenter d'expliquer les mécanismes qui conditionnent la compréhension des sens internes et externes à une communauté scientifique précise. Les signes, au sens large du terme (mots, images, illustrations, photos, etc.), peuvent ainsi être des miroirs, car tout se passe comme s'ils étaient dotés d'un mouvement propre, qui n'est certes pas indépendant de leur environnement, mais qui le transcende. Ils "exhume[nt] les effets de sens des images par simple attribution de sens" [6: 143]. Deux pôles principaux sont pris en considération: la sémiotique verbale et la sémiotique nonverbale.

La sémiotique verbale vise prioritairement les jeux de langage exercés par les producteurs et utilisateurs du droit. Tout d'abord, les législateurs usent de ce jeu de langage afin d'éviter de fixer définitivement le sens d'un terme. Ce privilège offre ainsi la possibilité d'interprétations multiples. Il s'agit donc de construire des lois structurées, à plusieurs étages. Elles deviennent ainsi des lois dynamiques, flexibles, évolutives qui peuvent se définir au pluriel. C'est la volonté non équivoque manifestée par le législateur. Ce jeu de langage impose à l'interprète un examen complet du processus afin de cadrer son discours aux différents cas d'espèce.

Cette fiction, ce manque volontaire de frontières réelles entre la logique sémiotique et la multiplicité des champs d'application conduit à une instabilité de discours communs entre communautés scientifiques. Mais ces conflits de 'voisinage' s'avèrent bénéfiques au langage juridique par le rôle important laissé à l'interprétation. C'est également à ce niveau, dans la multiplicité, que le discours peut s'avérer fragile, susceptible de rupture dans la compréhension de sens [5: 141-143]. Mais c'est aussi à ce niveau que se joue la bataille de la signification de ces nouvelles notions qui, pour être validées et légales, ont besoin d'un support législatif cohérent.

Cet espace de liberté de création n'est pas le résultat d'un malentendu ou d'un non aboutissement. Il prend appui sur le contenu sémantique du texte qui, par son organisation, l'insère dans un corpus général de réflexion: la doctrine du flou. Sa forme particulière situe par exemple le juge dans un espace décisionnel relativement ouvert. La doctrine du Flou est non seulement productrice d'identité sociale mais également source de cohésion entre les individus d'une même communauté scientifique. Bachelier rappelle que "la richesse d'un concept scientifique se mesure à sa puissance de déformation" [4: 1]. Cette flexibilité du concept devient une réalité vivante, "a kind of personification ... as if the Law were a living creature" [2: 399].

La sémiotique non-verbale est le pendant de la stratégie du discours juridique au sens où elle peut véhiculer un sens d'universalité. Ces signes non-verbaux sont intégrés volontairement dans la conception actuelle des moyens de circulation de l'information juridique. C'est une dimension-parfois ignorée-de la communication, mais ces signes constituent un assemblage original et unique en son genre d'éléments iconographiques juxtaposés pour les seuls besoins d'une transposition visuelle et/ou transcription iconographique des différents pouvoirs et institutions, 
des codes et usages en vigueur dans les cours de justice, voire d'une simplification assumée de la codification du discours juridique pour les utilisateurs du droit.

Comme le précise si bien Debray [3: 126], "ces Image[s sont] plus virale[s] que l'écrit, elle[s ont] le don capital de souder la communauté croyante". En conséquence, c'est un élément qui ajoute une valeur supplémentaire à un écrit, à un oral, sans pour autant porter préjudice aux valeurs immédiates et propres. Il fait office de charnière, de relais entre cette conscience collective qu'il induit et l'expression de l'objet qu'il représente, et a pour but de tracer le parcours de l'œil dans le champ d'une culture juridique spécifique.

C'est en tant qu'instruments structurés et structurants de communication et de connaissance que les "systèmes symboliques" remplissent leur fonction politique d'instruments d'imposition ou de légitimation de la domination, qui contribuent à assurer la domination d'une classe sur une autre (violence symbolique) en apportant le renfort de leur force propre aux rapports de force qui les fondent [1: 206]. Aussi, comme tout signe est le fruit d'un acquis d'une communauté et d'un pays, sa compréhension hors de cet espace peut s'avérer un handicap gênant.

La mise en oeuvre du discours juridique se met donc en place. Ces objets d'étude constituent ainsi des constructions sociales, culturelles et représentationnelles fortes mettant en avant la nature communicationnelle du Droit. Ces «réalités» du Droit observées peuvent permettre de montrer des «visages» particuliers du Droit à un instant $\mathrm{T}$ pour une culture juridique spécifique. C'est donc sur la base d'une construction particulière d'une identité juridique que se lisent les différentes missions de recherche et publication menées dans ce domaine particulier. Ces visages peuvent être vus comme des entités en perpétuel remaniement et mouvement, comme des constructions subjectives de signes qui évaluent des significations particulières, voire comme une communauté de culture juridique.

Cette première édition d'une thématique autour de «Law and Cartoons» permet de réunir des spécialistes capables d'analyser la culture visuelle du droit pour pouvoir apporter des éléments de réponses aux questions suivantes: Comment le sens juridique est construit, distribué et interprété? Quelles sont les compétences, les codes d'interprétation exigés pour appréhender la culture visuelle? C'est ainsi que les multiples aspects visuels, culturels, médiatiques et populaires du droit y seront analysés (see [7]).

\section{Références}

1. Bourdieu, Pierre. 2001. Langage et pouvoir symbolique. Paris: Editions Seuil.

2. Burns, and H.L.A. Hart. 1977. Jeremy Bentham: A comment on the commentaries and a fragment on government. Londres: Athlone Press.

3. Debray, R. 1992. Vie et mort de l'image. Paris: Gallimard.

4. Grawitz, M. 1993. Méthodes des sciences sociales. Paris: Dalloz.

5. Hocreitère, P. 2003. "Sécurité et insécurité juridiques après la loi Solidarité et Renouvellement urbains". In Revue Française de Droit Administratif, 141-153. Janvier-Février 2003.

6. Jeudy, H.P. 1989. Les ruses de la communication: l'euthanasie des sages. Paris: Plon.

7. Wagner, Anne, and Richard K. Sherwin (eds.). 2013. Law, culture and visual studies. The Netherlands: Springer. 